\begin{tabular}{|c|c|}
\hline \multicolumn{2}{|c|}{ Statistica Sinica Preprint No: SS-2016-0067.R2 } \\
\hline Title & $\begin{array}{l}\text { Invariance properties and statistical inference for circular } \\
\text { data }\end{array}$ \\
\hline Manuscript ID & SS-2016-0067.R2 \\
\hline URL & http://www.stat.sinica.edu.tw/statistica/ \\
\hline DOI & $10.5705 /$ ss.202016.0067 \\
\hline Complete List of Authors & Gianluca Mastrantonio \\
\hline & Giovanna Jona Lasinio \\
\hline & Antonello Maruotti and \\
\hline & Gianfranco Calise \\
\hline Corresponding Author & Gianluca Mastrantonio \\
\hline E-mail & gianluca.mastrantonio@polito.it \\
\hline \multicolumn{2}{|c|}{ Notice: Accepted version subject to English editing. } \\
\hline
\end{tabular}


Statistica Sinica

\title{
Invariance properties and statistical inference for circular data
}

\author{
Gianluca Mastrantonio \\ Department of Mathematical Science, Polytechnic University of Turin \\ gianluca.mastrantonio@polito.it \\ Giovanna Jona Lasinio \\ Department of Statistical Sciences, University of Rome "Sapienza" \\ giovanna.jonalasinio@uniroma1.it
}

Antonello Maruotti

Centre for Innovation and Leadership in Health Sciences, University of Southampton

Department of Economic, Political Sciences and Modern Languages, LUMSA

a.maruotti@lumsa.it

\section{Gianfranco Calise}

Department of Earth Science, University of Rome "Sapienza"

gianfranco.calise@uniroma1.it

Abstract: Statistical inference on the circle may strongly depend on the chosen

reference system. Here, we introduce necessary and sufficient conditions to avoid inferential problems and misinterpretation of parameter estimates for any circular distribution. The construction of invariant distributions, with respect to the reference system, is discussed by introducing specific properties. Numerical 
examples on artificial and real data are presented to corroborate and illustrate

theoretical results. Keywords: Circular data, Initial direction, Invariance, Ori-

entation

\section{Introduction}

Circular data (for a review see e.g. Lee, 2010) arise naturally in many scientific fields where observations are recorded as directions or angles. Such data are encountered in environmental science (Bulla et al., 2012; ?; Wang and Gelfand, 2014; Lagona et al., 2015a, b; Mastrantonio et al., 2015, 2016a,b), animal movements (Eckert et al., 2008, Langrock et al., 2012, 2014; McLellan et al., 2015; Maruotti et al., 2016; Maruotti, 2016), social science (Gill and Hangartner, 2010) and musicology (Lee and Ho, 2008). Standard techniques cannot be used to analyze circular data, mainly due to the circular geometry of the sample space (for details, see Section 2). Many ad-hoc methods and statistical techniques have been developed to analyze and understand circular data (Mardia, 1972; Fisher, 1996; Mardia and Jupp, 1999; Jammalamadaka and SenGupta, 2001; Pewsey et al., 2013), leading

to important probability distribution theory and inferential results.

Probability distributions for circular data often assume a general structure using the unit circle as support and having a closed-form density. How- 
ever, circular data have some specific features that should be taken into account in any analysis. Indeed, circular data have no designed zero (i.e. initial direction) or end; moreover, the designation of the natural orientation is arbitrary. Despite having tractable forms, the use of well-known circular distributions may lead to misleading inference if the issues of the initial direction and orientation are overlooked. Hence the principal objective of this article is to study the impact on statistical inference of overlooking changes in the reference system.

We define two properties for circular distributions: the invariance under changes of initial direction (ICID) and the invariance under changes of system orientation (ICO). We demonstrate that only a distribution holding the two aforementioned properties allows inference independent of the reference system. We give necessary and sufficient conditions that a distribution must satisfies to verify ICID and ICO, and we investigate the nature of existing circular distributions in order to check if the invariance properties hold. We also show that by introducing two additional parameters, accounting for changes in the reference system, an invariant circular distributions can be built from one that does not verify the ICID and ICO properties. 
This article is organized as follows. Section 2 introduces the notation used throughout the article and basic definitions on circular variables. It discusses the ICID and ICO properties and provides examples demonstrating the importance of those properties to avoid misleading inference. Section 3 deals with the construction of invariant distributions. Focusing on widely-used circular distributions, we present the main statistical properties of invariant distributions and we will stress the inferential issues of overlooking the initial direction and orientation in empirical analysis. Section 4 considers some numerical examples pointing out the issues in parameters interpretation and model fitting if the mentioned data features are ignored. Section 5 concludes with a summary of the main results and some concluding remarks. Extensive examples of the use of our proposal are reported in the supplementary material, where Appendix A illustrates analytical applications and Appendix B collects some more numerical examples.

\section{Invariance in circular distributions}

Let $\{\mathbb{S}, \mathcal{A}, P\}$ be a probability space, where the sample space $\mathbb{S}=\{(x, y)$ : $\left.x^{2}+y^{2}=1\right\}$ is the unit circle, $\mathcal{A}$ is the $\sigma$-algebra on $\mathbb{S}$ and $P: \mathbb{S} \rightarrow[0,1]$ is the normalized Lebesgue measure on the measurable space $\{\mathbb{S}, \mathcal{A}\}$. Let $\mathbb{D}$ 
be a subset of $\mathbb{R}$ such that its length is $2 \pi$, i.e. $\mathbb{D}=[a, b)$ with $b-a=2 \pi$.

Let us consider the measurable function $\Theta: \mathbb{S} \rightarrow \mathbb{D}$, with $\Theta^{-1}(d)=$ $(x, y)=(\cos d, \sin d)$, let $\mathcal{D}=\sigma(\mathbb{D})$ be the $\sigma$-algebra of $\mathbb{D}$ induced by $\Theta$, $A_{\Theta, D} \equiv\{(x, y): \Theta(x, y) \in D\}$ and $\mathbb{P}_{\Theta}(D)=P\left(\Theta^{-1}(D)\right)=P\left(A_{\Theta, D}\right), \forall D \in$ $\mathcal{D}$. Then the measurable space induced by $\Theta$ is $\left(\mathbb{D}, \mathcal{D}, \mathbb{P}_{\Theta}\right)$ with

1. $\mathbb{P}_{\Theta}(D)=P\left(A_{\Theta, D}\right) \geq 0, \forall D \in \mathcal{D}$;

2. $\mathbb{P}_{\Theta}(\mathbb{D})=P\left(A_{\Theta, \mathbb{D}}\right)=1$;

3. for any countable sequence of disjoint sets $\left\{D_{j}\right\}_{j=1}^{\infty}$ of $\mathcal{D}, \mathbb{P}_{\Theta}\left(\cup_{j=1}^{\infty} D_{j}\right)=$ $P\left(A_{\Theta, \cup_{j=1}^{\infty} D_{j}}\right)=P\left(\cup_{j=1}^{\infty} A_{\Theta, D_{j}}\right)=\sum_{j=1}^{\infty} P\left(A_{\Theta, D_{j}}\right)=\sum_{j=1}^{\infty} \mathbb{P}_{\Theta}\left(D_{j}\right)$,

i.e. $\left(\mathbb{D}, \mathcal{D}, \mathbb{P}_{\Theta}\right)$ is a probability space.

It follows that $\Theta$ is a circular random variable and $\mathbb{P}_{\Theta}$ is its probability distribution. Accordingly, for all $d \in \mathbb{D}, \Theta^{-1}(d)=\Theta^{-1}(d \bmod (2 \pi))$. $\mathbb{D}$ can be either continuous or discrete and, in the latter case, it is composed of $l<$ $\infty$ distinct points equally spaced between 0 and $2 \pi$ with $\mathbb{D} \equiv\{2 \pi j / l\}_{j=0}^{l-1}$. If $\mathbb{D}$ is a continuous domain, $\Theta$ is a continuous circular variable and $\mathbb{P}_{\Theta}$ is the Lebesgue measure; if $\mathbb{D}$ is discrete, $\Theta$ is a discrete circular variable and $\mathbb{P}_{\Theta}$ is its counting measure. In both cases, let $f_{\Theta}$ be the probability density function (pdf) of $\Theta$, with $f_{\Theta}=d \mathbb{P}_{\Theta} / d P_{\Theta}: \mathbb{D} \rightarrow \mathbb{R}^{+}$, i.e. $\mathbb{P}_{\Theta}(D)=$ $\int_{D} f_{\Theta} d P_{\Theta}$ 
In the representation of circular variables, a key role is played by the initial direction and the orientation of the domain (clockwise or anti-clockwise). Both are uniquely determined by the choice of the orthogonal reference system on the plane. Any statistical tool for circular variables should be invariant with respect to different choices of the reference system to avoid conflicting or misleading conclusions, see Section 3.3.

Let $\boldsymbol{\psi}$ be the vector of parameters, ICID and ICO distributions are defined as follow:

Definition 1. A probability density function $f_{\Theta}$ is ICID, i.e. invariant under changes of initial direction, if for all $\xi \in \mathbb{D}$ and $\boldsymbol{\psi} \in \boldsymbol{\Psi}$, there exists $\boldsymbol{\psi}^{*} \in \boldsymbol{\Psi}$ such that $f_{\Theta}(\theta \mid \boldsymbol{\psi})=f_{\Theta}\left(\theta-\xi \mid \boldsymbol{\psi}^{*}\right)$ for all $\theta \in \mathbb{D}$

Definition 2. A probability density function $f_{\Theta}$ is ICO, i.e. invariant under changes of the reference system orientation, if for all $\boldsymbol{\psi} \in \boldsymbol{\Psi}$, there exists $\boldsymbol{\psi}^{*} \in \boldsymbol{\Psi}$ such that $f_{\Theta}(\theta \mid \boldsymbol{\psi})=f_{\Theta}\left(-\theta \mid \boldsymbol{\psi}^{*}\right)$ for all $\theta \in \mathbb{D}$.

The following examples help to visualize definitions 1 and 2 and why inference should not depend on the reference system. In Figure 1 (a), a wrapped skew normal (WSN) density (Pewsey, 2000) is plotted. The origin (initial direction) is chosen according to a geographical template and set to East. The orientation is anti-clockwise. By changing the initial direction (Figure 1 (b)) or the system orientation (Figure 1(c)), we can obtain WSN 
Wrapped skew normal

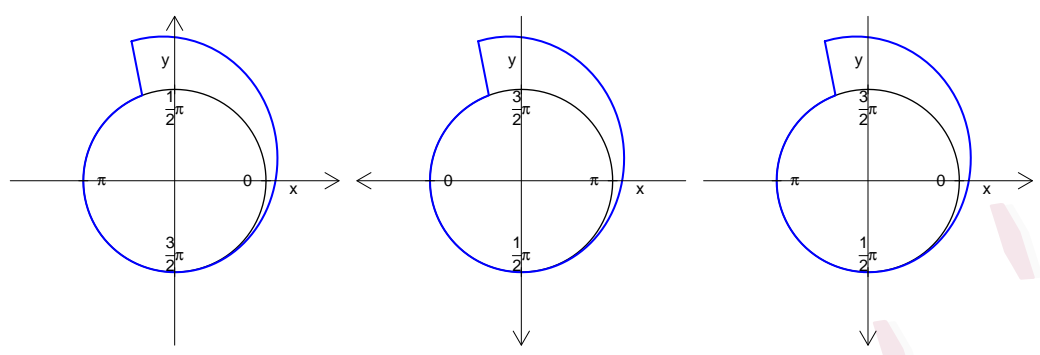

(a)

(b)

(c)

Wrapped exponential

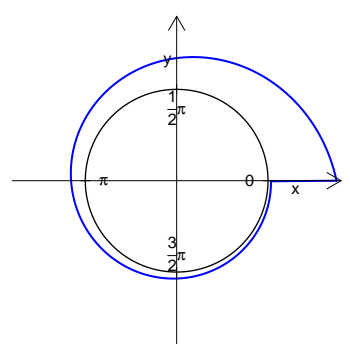

(d)

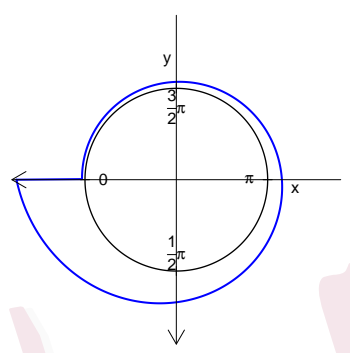

(e)

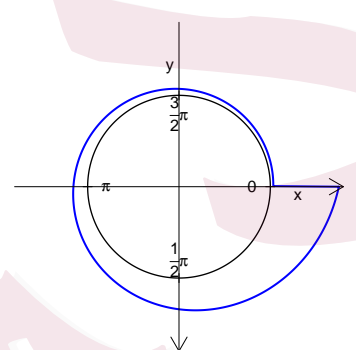

(f)

Figure 1: Probability density functions of a WSN (a-c) and a WE (d-f) under different initial directions and orientations. The arrows indicate the axis orientation

pdfs with shapes exactly as the one in Figure 1 (a), i.e. there exists $\boldsymbol{\psi}^{*}$ such that $f_{\Theta}(\theta \mid \boldsymbol{\psi})=f_{\Theta}\left(\theta-\pi \mid \boldsymbol{\psi}^{*}\right)$ (the ICID definition) and $f_{\Theta}(\theta \mid \boldsymbol{\psi})=$ $f_{\Theta}\left(-\theta \mid \boldsymbol{\psi}^{* *}\right)$ (the ICO definition). This example is not sufficient to prove that the WSN verifies the ICID and ICO because the properties must hold for all possible reference systems; the formal demonstration will be given in 
Section 3.3 using Theorem 1 that will be introduced in the next section.

A further example is provided in Figure 1 (d): a wrapped exponential (WE) distribution (Jammalamadaka and Kozubowski, 2004) is considered here. We proceed as before, changing the initial direction (Figure 1 (e)) and the system orientation (Figure 1 (f)). The intuition tells us that a necessary (but not sufficient) condition for a circular distribution to be ICID and ICO is that the circular variance must be constant across reference systems (technical details are given in Section 3.2). By investigating the WE, we can see that this is verified only by the densities in Figure 1 (e) and (f) that, on the other hand, concentrate their probability mass in different portion of the circle with respect to Figure 1 (d). Hence the wrapped exponential is not ICID nor ICO.

\section{Building invariant circular distributions}

In this section we introduce necessary and sufficient conditions for a distribution to verify ICID and ICO. Based on these conditions, we derive the ICID and ICO counterparts of existing distributions. 
3.1 Invariance: necessary and sufficient conditions

\subsection{Invariance: necessary and sufficient conditions}

Theorem 1. Let $f_{\Theta}(\cdot \mid \psi)$ be the pdf of the circular variable $\Theta \in \mathbb{D}$, with $\boldsymbol{\psi} \in \boldsymbol{\Psi}$. Let $\Theta^{*}=\delta(\Theta+\xi)$, with $\delta=\{-1,1\}$ and $\xi \in \mathbb{D}$ and $f_{\Theta^{*}}\left(\cdot \mid \boldsymbol{\psi}^{*}\right)$, $\boldsymbol{\psi}^{*} \in \Psi^{*}$. Then $f_{\Theta}$ is ICID and ICO iff $f_{\Theta^{*}}\left(\theta^{*} \mid \boldsymbol{\psi}^{*}\right)=f_{\Theta}\left(\theta^{*} \mid \boldsymbol{\psi}^{*}\right)$ everywhere with $\boldsymbol{\Psi}^{*} \equiv \boldsymbol{\Psi}$, i.e. $f_{\Theta}$ and $f_{\Theta^{*}}$ belong to the same parametric family.

Proof. From the rule of variable transformation we have that

$$
f_{\Theta^{*}}\left(\theta^{*} \mid \boldsymbol{\psi}^{*}\right)=f_{\Theta}\left(\delta \theta^{*}-\xi \mid \boldsymbol{\psi}\right)
$$

where $\boldsymbol{\psi}^{*}$ is a function of $(\boldsymbol{\psi}, \delta, \xi)$.

1. If $f_{\Theta^{*}}\left(\theta^{*} \mid \boldsymbol{\psi}^{*}\right)=f_{\Theta}\left(\theta^{*} \mid \boldsymbol{\psi}^{*}\right)$ then $f_{\Theta}$ is ICID and ICO

Equation (3.1) is true for all $\theta^{*} \in \mathbb{D}, \xi \in \mathbb{D}, \delta \in\{-1,1\}$. As long as $f_{\Theta^{*}}$ and $f_{\Theta}$ belong to the same parametric family it is $\Psi^{*} \equiv \boldsymbol{\Psi}$. Then we can write

$$
f_{\Theta}\left(\theta^{*} \mid \boldsymbol{\psi}^{*}\right)=f_{\Theta}\left(\delta \theta^{*}-\xi \mid \boldsymbol{\psi}\right)
$$

It is sufficient to set $\delta=1$ in equation $\sqrt{3.2}$ to satisfy Definition 1 and $\delta=-1$ and $\xi=0$ to satisfy Definition 2 .

2. If $f_{\Theta}$ is ICID and ICO then $f_{\Theta^{*}}\left(\theta^{*} \mid \boldsymbol{\psi}^{*}\right)=f_{\Theta}\left(\theta^{*} \mid \boldsymbol{\psi}^{*}\right)$. According to the ICID and ICO properties the following relation 
3.1 Invariance: necessary and sufficient conditions

holds: $f_{\Theta}\left(\delta \theta^{*}-\xi \mid \boldsymbol{\psi}\right)=f_{\Theta}\left(\theta^{*} \mid \boldsymbol{\psi}^{* *}\right)$, where $\boldsymbol{\psi}^{* *} \in \boldsymbol{\Psi}$. Since $f_{\Theta^{*}}\left(\theta^{*} \mid \boldsymbol{\psi}^{*}\right)=$ $f_{\Theta}\left(\delta \theta^{*}-\xi \mid \boldsymbol{\psi}\right)$ (equation $\left.(3.1)\right)$ we have $f_{\Theta^{*}}\left(\theta^{*} \mid \boldsymbol{\psi}^{*}\right)=f_{\Theta}\left(\theta^{*} \mid \boldsymbol{\psi}^{* *}\right)$ that implies $\boldsymbol{\psi}^{*} \equiv \boldsymbol{\psi}^{* *}$, ending the proof.

It is always possible to transform any non invariant pdf so to obtain its ICID and ICO version.

Proposition 1. If $f_{\Theta}(\cdot \mid \boldsymbol{\psi})$ is not ICID and ICO, the density $f_{\Theta^{*}}\left(\cdot \mid \boldsymbol{\psi}^{*}\right)$, where $\Theta^{*}=\delta(\Theta+\xi), \delta \in\{-1,1\}, \xi \in \mathbb{D}$ and $\boldsymbol{\psi}^{*}=(\boldsymbol{\psi}, \delta, \xi) \in \boldsymbol{\Psi}^{*}$, is ICID and $I C O$.

Proof. Let $\Theta^{* *}=\delta^{*}\left(\Theta^{*}+\xi^{*}\right)$. Following Theorem 1, if $f_{\Theta^{* *}}$ belongs to the same parametric family as $f_{\Theta^{*}}$, then $f_{\Theta^{*}}$ is ICID and ICO. Because $f_{\Theta^{* *}}\left(\theta^{* *} \mid \boldsymbol{\psi}^{* *}\right)=f_{\Theta^{*}}\left(\delta^{*} \theta^{* *}-\xi^{*} \mid \boldsymbol{\psi}^{*}\right)$ and $f_{\Theta^{*}}\left(\theta^{*} \mid \boldsymbol{\psi}^{*}\right)=f_{\Theta}\left(\delta \theta^{*}-\xi \mid \boldsymbol{\psi}\right)$, the following identities hold: $f_{\Theta^{* *}}\left(\theta^{* *} \mid \boldsymbol{\psi}^{* *}\right)=f_{\Theta^{*}}\left(\delta^{*} \theta^{* *}-\xi^{*} \mid \boldsymbol{\psi}^{*}\right)=f_{\Theta}\left(\delta\left(\delta^{*} \theta^{* *}-\right.\right.$ $\left.\left.\xi^{*}\right)-\xi \mid \boldsymbol{\psi}\right)$. Now let $\delta^{* *}=\delta \delta^{*}$ and $\xi^{* *}=\left(\delta \xi^{*}+\xi\right)$ we can write $f_{\Theta^{* *}}\left(\theta^{* *} \mid \boldsymbol{\psi}^{* *}\right)=$ $f_{\Theta}\left(\delta^{* *} \theta^{* *}-\xi^{* *} \mid \boldsymbol{\psi}\right)$. Bearing in mind that $\delta^{* *} \in\{-1,1\}$ and $\xi^{* *} \in \mathbb{D}, \Theta^{* *}$ is obtained starting from $\Theta$ by transforming $\Theta^{*}$ and the vector of parameters $\left(\boldsymbol{\psi}, \delta^{* *}, \xi^{* *}\right)$ belonging to the same space $\boldsymbol{\Psi}^{*}$ of $\boldsymbol{\psi}^{*}=(\boldsymbol{\psi}, \delta, \xi)$. Then $f_{\Theta^{* *}}$ and $f_{\Theta^{*}}$ belong to the same parametric family. 
3.2 Invariance: statistical properties

By applying the previous proposition, we get the invariant version of any circular density.

\subsection{Invariance: statistical properties}

A wide number of circular distributions have been extensively studied in the literature, and their characteristic functions, as well as the trigonometric moments, circular means and concentrations have been defined. Here we show how to obtain these quantities when ICID and ICO distributions are obtained by using Proposition 1 .

The trigonometric moments, $\alpha_{p}=E \cos p \Theta$ and $\beta_{p}=E \sin p \Theta$, of a circular density are related to the characteristic function $\varphi_{\Theta}(p)$ of $\Theta$ : $\varphi_{\Theta}(p)=E(\exp (i p \Theta))=\alpha_{p}+i \beta_{p}$. Let $c_{p}=\left|\varphi_{\Theta}(p)\right|=\sqrt{\alpha_{p}^{2}+\beta_{p}^{2}}$ and $\mu_{p}=\operatorname{atan}^{*}\left(\beta_{p} / \alpha_{p}\right)$ (the function atan* is the modified inverse tangent function, see for example Jammalamadaka and SenGupta (2001)). It is well known that $\varphi_{\Theta}(p)=c_{p} \exp \left(i \mu_{p}\right)=c_{p} \cos \mu_{p}+i c_{p} \sin \mu_{p}$, and then $\alpha_{p}=c_{p} \cos \mu_{p}$ and $\beta_{p}=c_{p} \sin \mu_{p}$. When $p=1$, the quantities $\mu_{1}$ and $c_{1}$ are called circular mean and circular concentration respectively. Now let $\Theta^{*}=\delta(\Theta+\xi)$ and suppose that the density of $\Theta$ is not invariant. Following Proposition 1, the density of $\Theta^{*}$ is ICID and ICO. $\Theta^{*}$ is a linear 
transformation of $\Theta$ and its characteristic function is then

$$
\varphi_{\Theta^{*}}(p)=e^{i p \delta \xi} \varphi_{\Theta}(\delta p)=c_{\delta p} e^{i\left(p \delta \xi+\mu_{\delta p}\right)} .
$$

$\varphi_{\Theta}(-p)$ is the complex conjugate of $\varphi_{\Theta}(p)$ and since $\left|\varphi_{\Theta}(-p)\right|=\left|\varphi_{\Theta}(p)\right|$ then $c_{p}=c_{-p}, \alpha_{-p}=\alpha_{p}, \beta_{-p}=-\beta_{p}$ and it follows that $\mu_{-p}=-\mu_{p}$. Then equation 3.3 can be written as $c_{p} \exp \left(i\left(p \delta \xi+\delta \mu_{p}\right)\right)=c_{p} \cos \left(p \delta \xi+\delta \mu_{p}\right)+$ $i c_{p} \sin \left(p \delta \xi+\delta \mu_{p}\right)$. Let $\alpha_{p}^{*}, \beta_{p}^{*}, \mu_{p}^{*}$ and $c_{p}^{*}$ be the trigonometric moments, the circular mean and concentration of the random variable $\Theta^{*}$, we have that

$$
\begin{aligned}
& \alpha_{p}^{*}=c_{p} \cos \left(p \delta \xi+\delta \mu_{p}\right)=c_{p} \cos \left(p \xi+\mu_{p}\right), \\
& \beta_{p}^{*}=c_{p} \sin \left(p \delta \xi+\delta \mu_{p}\right)=\delta c_{p} \sin \left(p \xi+\mu_{p}\right),
\end{aligned}
$$

with $\mu_{p}^{*}=p \delta \xi+\delta \mu_{p}$ and $c_{p}^{*}=c_{p}$. Notice that, for all invariant distributions, equations 3.4 and 3.5 can be used to compute the trigonometric moments when the reference system is changed.

\subsection{Examples of inferential problems}

Let us consider a $\mathrm{WE}(\lambda)$ circular variable $\Theta$, first of all we verify if this distribution is ICID and ICO. The WE (Jammalamadaka and Kozubowski, 2004) has density

$$
f_{\Theta}(\theta \mid \lambda)=\frac{\lambda e^{-\lambda \theta}}{1-e^{-2 \pi \lambda}}, \lambda>0
$$




\subsection{Examples of inferential problems}

We find the density of the random variable $\Theta^{*}$ that is

$$
f_{\Theta^{*}}\left(\theta^{*} \mid \lambda, \delta, \xi\right)=\frac{\lambda e^{-\lambda\left(\delta \theta^{*}-\xi\right)}}{1-e^{-2 \pi \lambda}}
$$

To see that the WE is not ICID and ICO, it is sufficient to prove that for a given $\lambda, \delta$ and $\xi$, does not exist a $\lambda^{*}$ such that $(3.7)$ can be written as a WE density, i.e. the following equality does not hold

$$
\frac{\lambda e^{-\lambda\left(\delta \theta^{*}-\xi\right)}}{1-e^{-2 \pi \lambda}}=\frac{\lambda^{*} e^{-\lambda^{*}\left(\theta^{*} \bmod (2 \pi)\right)}}{1-e^{-2 \pi \lambda^{*}}} .
$$

In $(3.8)$ the modulus on the right side is required to ensure that the density is well defined. To show that the WE is not ICID and ICO, it is sufficient to show that $-\lambda\left(\delta \theta^{*}-\xi\right) \neq-\lambda^{*}\left(\theta^{*} \bmod (2 \pi)\right)$ for a set of values. For example, at $\Theta^{*}=2 \pi$, with $\delta=1$, we obtaining $-\lambda(2 \pi-\xi)=-\lambda^{*} 0$; thus, the WE is not ICID and ICO. Notice that the density of $\Theta^{*}$ in equation (3.7) is the invariant version of the wrapped exponential (IWE) and it depends on parameters $\lambda, \delta, \xi$. The domain of $\Theta^{*}$ depends on $\delta$ and $\xi$ and this may lead to issues in model fitting, then we prefer to write 3.7 as

$$
f_{\Theta^{*}}\left(\theta^{*} \mid \lambda, \delta, \xi\right)=\frac{\lambda e^{-\lambda\left[\left(\delta \theta^{*}-\xi\right) \bmod (2 \pi)\right]}}{1-e^{-2 \pi \lambda}}, \theta^{*} \in \mathbb{D} .
$$

The trigonometric moments of the WE (see Jammalamadaka and Kozubowski, $2004)$ are $\lambda / \sqrt{\lambda^{2}+p^{2}} \cos \left(\operatorname{atan}^{*}(p / \lambda)\right)$ and $\lambda / \sqrt{\lambda^{2}+p^{2}} \sin \left(\operatorname{atan}^{*}(p / \lambda)\right)$ and from them, using the results of Section 3.2, we can derive the ones of the 
3.3 Examples of inferential problems

IWE; $\alpha_{p}=\frac{\lambda}{\sqrt{\lambda^{2}+p^{2}}} \cos \left(p \xi+\operatorname{atan}^{*} \frac{p}{\lambda}\right)$ and $\beta_{p}=\delta \frac{\lambda}{\sqrt{\lambda^{2}+p^{2}}} \sin \left(p \xi+\operatorname{atan}^{*} \frac{p}{\lambda}\right)$. The circular mean is then $\mu_{1}=\delta \xi+\delta \operatorname{atan}^{*}(1 / \lambda)$ and the circular concentration is $c_{1}=\lambda / \sqrt{\lambda^{2}+1^{2}}$.

Now consider $n$ observations $\theta_{1}, \ldots \theta_{n}$ WE distributed, in a reference system with zero direction set to North and clockwise orientation. We are interested in finding the maximum likelihood estimator (MLE) of $\lambda$, i.e. $\hat{\lambda}$. Notice that the WE density, equation (3.6), has the same functional form of the truncated exponential defined over the domain $[0,2 \pi)$. Then, likelihood functions based on the WE or the truncated exponential lead to the same MLE. For a truncated exponential likelihood over a given interval $[a, b)$, the MLE of $\lambda$ exists only if the arithmetic mean of the observations is smaller than $(b+a) / 2$ (Walter L. Deemer, 1955), then, in our setting $\hat{\lambda}$ is defined only if $\sum \theta_{i} / n<\pi$. Let us assume that we observed the same circular variable but we recorded its values using an anticlockwise orientation. The data have the same nature as before, they represent the same phenomenon and we just changed the reference system. In the new reference system the values of the circular variables become $2 \pi-\theta_{i}$ and the MLE of $\lambda$ exists only if $2 \pi-\sum \theta_{i} / n<\pi \Rightarrow \sum \theta_{i} / n>\pi$. The two conditions for the existence of $\hat{\lambda}$ in the two reference systems, i.e. $\sum \theta_{i} / n<\pi$ and $\sum \theta_{i} / n>\pi$, cannot be verified at the same time. It follows that the inference with the WE 


\subsection{Examples of inferential problems}

depends on the choice of the reference system orientation.

For the same phenomenon, we record the values of the circular variable in a new reference system, where the zero direction is now set to be the angle $d \in[0,2 \pi)$ of the first system; the observed circular variable's values become $\theta_{i}-d$. Let suppose $d \geq \max _{i, i=1, \ldots, n} \theta_{i}$, and then $\theta_{i}-d=$ $2 \pi+\theta_{i}-d$. In this new reference system, the condition for the existence of $\hat{\lambda}$ is $2 \pi+\sum \theta_{i} / n-d<\pi \Rightarrow \sum \theta_{i} / n<d-\pi$. If $d=\pi$, the MLE does not exist.

On the other hand, the MLEs of $\lambda, \delta$ and $\xi$, under an IWE likelihood, always exists. $\hat{\lambda}$, in the original reference system, exists if $\sum\left[\left(\delta \theta_{i}-\xi\right) \bmod (2 \pi)\right] / n<$ $\pi$ holds, that is at least when $\delta=1$ and $\xi=0$ if $\sum \theta_{i} / n<\pi$ and $\delta=-1$ and $\xi=\epsilon$ otherwise where $\epsilon \in\left(0, \min _{i, i=1, \ldots, n} \theta_{i}\right)$. In the second reference system (anticlockwise orientation) the condition becomes $\sum\left[\left(\delta 2 \pi-\delta \theta_{i}-\xi\right) \bmod (2 \pi)\right] / n<\pi$ that is verified at least when $\delta=-1$ and $\xi=0$ if $\sum \theta_{i} / n<\pi$ and $\delta=1$ and $\xi=\epsilon$ otherwise. To conclude, In the third reference system (changed zero direction) the existence condition is $\sum\left[\left(\delta \theta_{i}-\delta d-\xi\right) \bmod (2 \pi)\right] / n<\pi$ that is verified with $\delta=1$ and $\xi=2 \pi-d$ if $\sum \theta_{i} / n<\pi$ and $\delta=-1$ and $\xi=d+\epsilon$ otherwise. We are not able to find a closed form for the MLE of $\delta$ and $\xi$ but the log-likelihood is proportional to $-\sum\left[\left(\delta \theta_{i}^{*}-\xi\right)\right]$ that is a finite function of $(\delta, \xi)$ and this is 


\subsection{Examples of inferential problems}

a sufficient condition for the existence of their MLEs.

As a further example, let us now consider a discrete circular variable $\Theta$ that can assume $l$ equally spaced points on the circle, such variable is said to follow a wrapped Poisson (WP) distribution with parameter $\lambda>0$ (Mardia and Jupp, 1999), i.e. $\Theta \sim W P_{l}(\lambda)$, if it has pdf $\sum_{k=0}^{\infty} \lambda^{\theta \frac{l}{2 \pi}+k l} e^{-\lambda} /(\theta l / 2 \pi+k l)$ !. The WP is not ICID and ICO (see the Supplementary material for details) but, using Proposition 1, we can obtain its invariant invariant version (IWP), that has density

$$
f_{\Theta^{*}}\left(\theta^{*} \mid \lambda, \delta, \xi\right)=\sum_{k=0}^{\infty} \frac{\lambda^{\left[\left(\delta \theta^{*}-\xi\right) \bmod (2 \pi)\right] \frac{l}{2 \pi}+k l} e^{-\lambda}}{\left(\left[\left(\delta \theta^{*}-\xi\right) \bmod (2 \pi)\right] \frac{l}{2 \pi}+k l\right) !}, \theta^{*} \in \mathbb{D} .
$$

Now let $\Theta \sim W P_{l}(\lambda)$ with $n$ samples $\theta_{i}$, in a reference system with zero direction North and clockwise orientation. Let $\hat{\lambda}$ be the MLE of $\lambda$ and $\hat{\mu}_{1}$ and $\hat{c}_{1}$ the associated circular mean and concentration, that are respectively $\mu_{1}=\lambda \sin (2 \pi / l)$ and $c_{1}=\exp (-\lambda(1-\cos (2 \pi / l)))$. If we change orientation, in the new reference system the circular observations become $2 \pi-\theta_{i}$. Since the transformation $2 \pi-\theta_{i}$ is linear and the data are the same as in the first reference system, the circular concentration should remain the same, i.e. $\hat{c}_{1}^{*}=\hat{c}_{1}$, while the circular mean will change according to the transformation, i.e. $\hat{\mu}_{1}^{*}=2 \pi-\hat{\mu}_{1}$. Since $\hat{c}_{1}=\exp (-\hat{\lambda}(1-\cos (2 \pi / l)))$, then $\hat{c}_{1}^{*}=\hat{c}_{1}$ only if $\hat{\lambda}^{*}=\hat{\lambda}$ and it follows that the equivalence $\hat{\mu}_{1}^{*}=2 \pi-\hat{\mu}_{1}$ is never verified, i.e. $\hat{\lambda}^{*} \sin (2 \pi / l) \neq 2 \pi-\hat{\lambda} \sin (2 \pi / l)$ if $\hat{\lambda}^{*}=\hat{\lambda}$. 
If we set to $d \in[0,2 \pi)$ the new zero direction, the circular observations become $\theta_{i}-d$. Here again we expect $\hat{c}_{1}^{*}=\hat{c}_{1}$ while $\hat{\mu}_{1}^{*}=\hat{\mu}_{1}-d$. Since the two circular concentrations should be the same, then $\hat{\lambda}^{*}=\hat{\lambda}$ and again the equivalence $\hat{\lambda}^{*} \sin (2 \pi / l)=\hat{\lambda} \sin (2 \pi / l)-d$ is never verified if $d>0$. On the other hand the circular mean of any invariant density can be written as $\delta \xi+\delta \mu_{1}$, where we can think of $\mu_{1}$ as a "baseline" circular mean, and parameters $\delta$ and $\xi$ can account for changes in the reference system in a coherent way without affecting the circular concentration, see Section 3.2 .

\section{$4 \quad$ Numerical examples}

Two examples are considered in this section. The first is based on simulated continuous data and the other is a real life problem where discrete circular variables were observed. Our intent lies in highlighting the consequences of the lack of ICO and ICID properties on model inference. We use three reference systems: the first fixes North as zero direction and chooses a clockwise orientation (RS1), in the second reference system (RS2) we move the zero direction to East, while we obtain the third reference system (RS3) by changing the orientation of $\mathrm{RS} 1$. In each reference system, we find the MLEs of the invariant distribution parameters, that are the IWE modeling 
continuous data and the IWP describing discrete data, and the MLE of the corresponding non invariant distributions parameters, (i.e. the WE with continuous data and WP for discrete data), together with their circular means and concentrations. We indicate with $\hat{\lambda}_{i}, \hat{\mu}_{1, i}$ and $\hat{c}_{1, i}$ the MLEs of the non invariant distribution parameter, circular mean and concentration in the $i^{\text {th }}$ reference system, respectively, while $\hat{\lambda}_{i}^{*}, \hat{\delta}_{i}^{*}, \hat{\xi}_{i}^{*}, \hat{\mu}_{1, i}^{*}$ and $\hat{c}_{1, i}^{*}$ are the parameters of the invariant distribution, circular mean and concentration in the $i^{\text {th }}$ reference system. The MLEs are found using numerical optimization procedures. Notice that an exact evaluation of the IWP density is not possible since it involves the evaluation of an infinite sum (see equation (3.9) ) and a truncation strategy, often used with wrapped distributions (see for example Coles and Casson, 1998; Jona Lasinio et al., 2012), is adopted; in detail we approximate $(3.9)$ with

$$
f_{\Theta^{*}}\left(\theta^{*} \mid \lambda, \delta, \xi\right)=\sum_{k=0}^{k_{\max }} \frac{\lambda^{\left[\left(\delta \theta^{*}-\xi\right) \bmod (2 \pi)\right] \frac{l}{2 \pi}+k l} e^{-\lambda}}{\left(\left[\left(\delta \theta^{*}-\xi\right) \bmod (2 \pi)\right] \frac{l}{2 \pi}+k l\right) !}, \theta^{*} \in \mathbb{D},
$$

and we choose $k_{\max }$ so that the total probability mass captured by the approximation is $>0.99999$.

Artificial data - Wrapped exponential We simulate 500 observations from a $W E(1)$ in the RS1. The MLEs of the WE and IWE parameters are shown in Table 1 while Figure 2 illustrates the real density used to simulate 


\begin{tabular}{c|ccc|cccccc}
\hline & \multicolumn{3}{|c|}{ WE } & \multicolumn{5}{c}{ IWE } \\
\hline \hline & $\hat{\lambda}_{i}$ & $\hat{\mu}_{1, i}$ & $\hat{c}_{1, i}$ & $\hat{\lambda}_{i}^{*}$ & $\hat{\delta}_{i}^{*}$ & $\hat{\xi}_{i}^{*}$ & $\hat{\mu}_{1, i}^{*}$ & $\hat{c}_{1, i}^{*}$ \\
\hline $\mathrm{RS} 1$ & 1.04 & 0.7658 & 0.7208 & 1.04 & 1 & 0 & 0.7658 & 0.7208 \\
$\mathrm{RS} 2$ & $\cdot$ & $\cdot$ & $\cdot$ & 1.04 & 1 & 4.7124 & 5.4782 & 0.7208 \\
$\mathrm{RS} 3$ & $\cdot$ & $\cdot$ & $\cdot$ & 1.04 & -1 & 0 & 5.5174 & 0.7208 \\
\hline \hline
\end{tabular}

Table 1: Simulated example - MLE of the WE and IWE parameters, circular mean and concentration

the data and the WE and IWE densities obtained with the MLEs in the three reference systems.

Real data - Wind direction The wind direction data are recorded on January 2000 at the monitoring station of Capo Palinuro (South Italy). The monitoring station of Capo Palinuro (WMO code 16310) is one of the coastal stations managed by the Meteorological Service of the Italian Air Force. The station is located on the rocky cape of Capo Palinuro, in the town of Centola in the province of Salerno, South Italy. Wind directions are monitored and routinely collected by several environmental agencies. Analyzed data come from reports prepared at the station and provided by the National Center of Aeronautical Meteorology and Climatology 


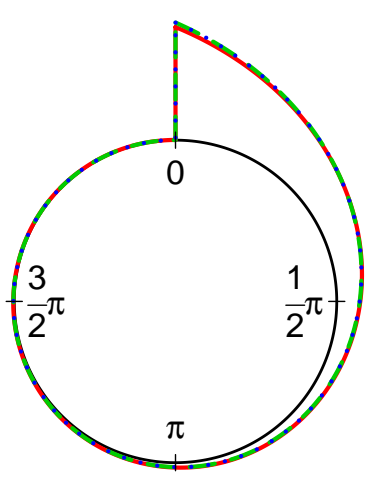

(a) RS1

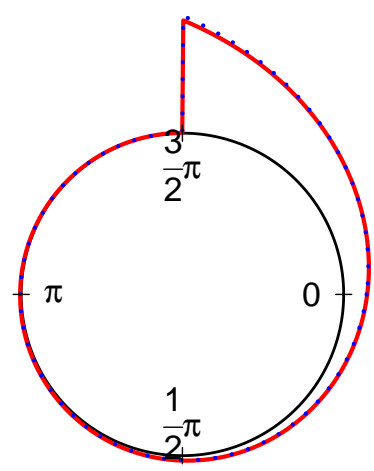

(b) RS2

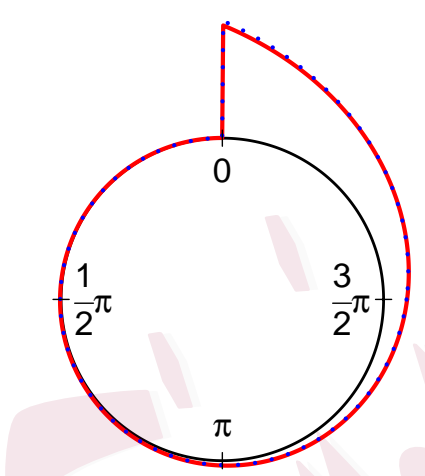

(c) RS3

Figure 2: Simulated example - Density used to simulate the data (solid line), wrapped exponential (dashed line) and invariant wrapped exponential (dotted line) density computed using the MLE of the parameters in the three reference systems (the WE MLE does not exist in RS2 and RS3).

(C.N.M.C.A.), special office of the Meteorological Service of the Italian Air Force. The database includes date and time of registration, direction of the wind in degrees, with eight daily measurements (every three hours) in the month of January 2000, i.e. we have 240 observations. The measuring instrument, anemometer, is placed away from obstacles and at an height of 10 meters above ground. A relevant issue with this measurement instrument is that it measures wind directions on a discrete scale dividing the circle 


\begin{tabular}{c|ccc|cccccc}
\hline & \multicolumn{3}{c|}{ WP } & \multicolumn{7}{c}{ IWP } \\
\hline \hline & $\hat{\lambda}_{i}$ & $\hat{\mu}_{1, i}$ & $\hat{c}_{1, i}$ & $\hat{\lambda}_{i}^{*}$ & $\hat{\delta}_{i}^{*}$ & $\hat{\xi}_{i}^{*}$ & $\hat{\mu}_{1, i}^{*}$ & $\hat{c}_{1, i}^{*}$ \\
\hline $\mathrm{RS} 1$ & 37.3434 & 0.2014 & 0.567 & 50.1254 & 1 & 4.0143 & 0.1520 & 0.4657 \\
$\mathrm{RS} 2$ & 64.1283 & 4.8526 & 0.3775 & 50.1254 & 1 & 2.4435 & 4.8644 & 0.4657 \\
$\mathrm{RS} 3$ & 71.3427 & 12.3885 & 0.3383 & 50.1254 & -1 & 4.0143 & 6.1312 & 0.4657 \\
\hline \hline
\end{tabular}

Table 2: Wind example - MLE of the WP and IWP parameters, circular mean and circular concentration

into ten-degrees intervals $(l=36)$. The MLEs are reported in Table 2 while the barplot of the observed data and the WP and IWP densities obtained with the MLEs in the three reference systems, are shown in Figure 3.

General Comments The arithmetic mean $\sum \theta_{i} / n$ in the first example is equal to 0.9523 in the RS1, 4.4710 in the RS2 and 5.3308 in the RS3 and then, keeping in mind the results of Section 3.3, it is not surprising that the MLE of the WE parameter can be estimated only in the RS1.

In all the examples, see Table 1 and 2, we can appreciate how the MLEs of the invariant densities parameters are coherent in moving among the three reference systems. More precisely $\hat{\lambda}_{1}^{*}=\hat{\lambda}_{2}^{*}=\hat{\lambda}_{3}^{*}$, i.e. the MLE of $\lambda^{*}$ is unaffected by changes of orientation or zero direction, we have $\left(\hat{\delta}_{2}^{*}, \hat{\xi}_{2}^{*}\right)=$ 


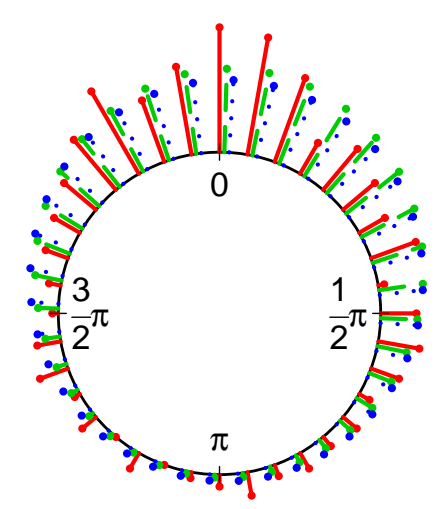

(a) RS1

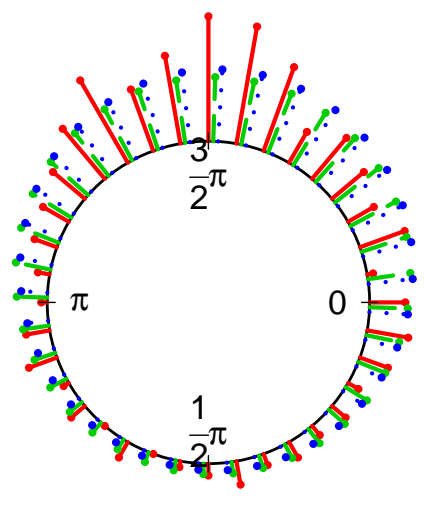

(b) RS2

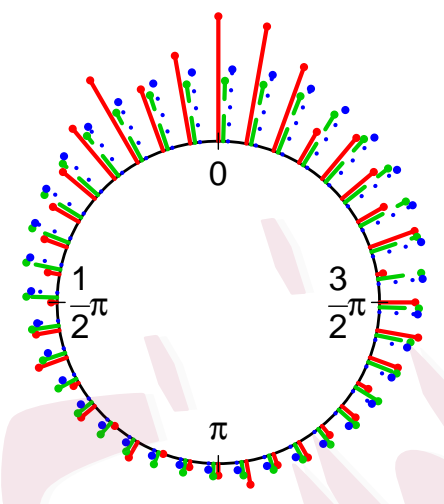

(c) RS3

Figure 3: Wind example - Density estimate of observed data (solid line), wrapped Poisson (dashed line) and invariant wrapped Poisson (dotted line) density computed using the MLE of the parameters in the three reference systems

$\left(\hat{\delta}_{1}^{*}, \hat{\xi}_{1}^{*}-\pi / 2\right)$ since in the RS2 we change the zero direction to $\pi / 2$, and in the RS3, where we modify the orientation, we have $\left(\hat{\delta}_{3}^{*}, \hat{\xi}_{3}^{*}\right)=\left(-\hat{\delta}_{1}^{*}, \hat{\xi}_{1}^{*}\right)$. Moreover the circular concentration remains the same in the three reference systems, i.e. $\hat{c}_{1,1}^{*}=\hat{c}_{1,2}^{*}=\hat{c}_{1,3}^{*}$, while the circular mean changes according to the reference system, i.e. $\hat{\mu}_{1,2}^{*}=\hat{\mu}_{1,1}^{*}-\pi / 2$ and $\hat{\mu}_{1,3}^{*}=-\hat{\mu}_{1,2}^{*}$. For the non invariant densities, even the circular concentration changes with the reference system as well as the MLE of the parameter $\lambda$ and the circular 
mean. The shapes of the invariant densities remain the same in the three reference systems, see Figures from 2 to 3.

\section{$5 \quad$ Summary and concluding remarks}

In this paper we formally introduced two properties, i.e. the invariance under changes of initial direction and the invariance under changes of the reference system orientation, that any circular distribution should hold to avoid misleading inferential results. We introduced the necessary and sufficient conditions that a circular distribution must satisfy to verify these two properties (ICID and ICO). By considering simulated and real data examples, we illustrated how misleading the use of not invariant distributions can be. We showed that not all the circular distributions proposed in the literature satisfy ICID and ICO properties and we developed a method to build ICID and ICO circular distributions. Trigonometric moments are easily obtained starting from those of the non invariant version of circular distributions (see Supplementary Material for more details and examples). Further use of the invariant distributions in complex models can be found in Mastrantonio and Calise (2016), where it is extended, in a Bayesian framework, to address classification issues with discrete circular and linear 


\section{REFERENCES}

variables; in the paper a hidden Markov model for discrete valued time series with linear and circular components is introduced. We believe that our proposal opens all new possibilities for the practical use of circular information measured on any scale.

\section{Acknowledgements}

This work is partially developed under the PRIN2015 supported-project "Environmental processes and human activities: capturing their interactions via statistical methods (EPHASTAT)" funded by MIUR (Italian Ministry of Education, University and Scientific Research).

\section{References}

Bulla, J., Lagona, F., Maruotti, A., and Picone, M. (2012). A multivariate hidden Markov model for the identification of sea regimes from incomplete skewed and circular time series. Journal of Agricultural, Biological, and Environmental Statistics, 17(4), 544-567.

Coles, S. and Casson, E. (1998). Extreme value modelling of hurricane wind speeds. Structural Safety, 20(3), $283-296$. 


\section{REFERENCES}

Eckert, S. A., Moore, J. E., Dunn, D. C., van Buiten, R. S., Eckert, K. L., and Halpin, P. N. (2008). Modeling loggerhead turtle movement in the mediterranean: importance of body size and oceanography. Ecological Applications, 18(2), 290-308.

Fisher, N. I. (1996). Statistical Analysis of Circular Data. Cambridge University Press, Cambridge.

Gill, J. and Hangartner, D. (2010). Circular data in political science and how to handle it. Political Analysis, 18(3), 316-336.

Jammalamadaka, S. R. and Kozubowski, T. J. (2004). New families of wrapped distributions for modeling skew circular data. Communications in Statistics - Theory and Methods, 33(9), 2059-2074.

Jammalamadaka, S. R. and SenGupta, A. (2001). Topics in Circular Statistics. World Scientific, Singapore.

Jona Lasinio, G., Gelfand, A., and Jona Lasinio, M. (2012). Spatial analysis of wave direction data using wrapped Gaussian processes. Annals of Applied Statistics, 6(4), 1478-1498.

Lagona, F., Picone, M., Maruotti, A., and Cosoli, S. (2015a). A hidden Markov approach to the analysis of space-time environmental data with 


\section{REFERENCES}

linear and circular components. Stochastic Environmental Research and Risk Assessment, 29(2), 397-409.

Lagona, F., Picone, M., and Maruotti, A. (2015b). A hidden Markov model for the analysis of cylindrical time series. Environmetrics, 26(8), 534-544. env.2355.

Langrock, R., King, R., Matthiopoulos, J., Thomas, L., Fortin, D., and Morales, J. M. (2012). Flexible and practical modeling of animal telemetry data: hidden Markov models and extensions. Ecology, 93(11), 23362342.

Langrock, R., Hopcraft, G., Blackwell, P., Goodall, V., King, R., Niu, M., Patterson, T., Pedersen, M., Skarin, A., and Schick, R. (2014). Modelling group dynamic animal movement. Methods in Ecology and Evolution, 5(2), 190-199.

Lee, A. (2010). Circular Data. Wiley Interdisciplinary Reviews: Computational Statistics, 2(4), 477-486.

Lee, J.-R. and Ho, H.-S. (2008). Circular statistics in musicology. Communicationsof the Korean Statistical Society, 15(2), 273-282. 


\section{REFERENCES}

Mardia, K. V. (1972). Statistics of Directional Data. Academic Press, London.

Mardia, K. V. and Jupp, P. E. (1999). Directional Statistics. John Wiley and Sons, Chichester.

Maruotti, A. (2016). Analyzing longitudinal circular data by projected normal models: a semi-parametric approach based on finite mixture models. Environmental and Ecological Statistics, 23(2), 257-277.

Maruotti, A., Punzo, A., Mastrantonio, G., and Lagona, F. (2016). A time-dependent extension of the projected normal regression model for longitudinal circular data based on a hidden Markov heterogeneity structure. Stochastic Environmental Research and Risk Assessment, 30(6), $1725-1740$.

Mastrantonio, G. and Calise, G. (2016). Hidden Markov model for discrete circularlinear wind data time series. Journal of Statistical Computation and Simulation, 86(13), 2611-2624.

Mastrantonio, G., Maruotti, A., and Jona Lasinio, G. (2015). Bayesian hidden Markov modelling using circular-linear general projected normal distribution. Environmetrics, 26, 145-158. 


\section{REFERENCES}

Mastrantonio, G., Jona Lasinio, G., and Gelfand, A. E. (2016a). Spatio-temporal circular models with non-separable covariance structure. TEST, 25(2), 331-350.

Mastrantonio, G., Gelfand, A. E., and Jona Lasinio, G. (2016b). The wrapped skew Gaussian process for analyzing spatio-temporal data. Stochastic Environmental Research and Risk Assessment, To appear.

McLellan, C. R., Worton, B. J., Deasy, W., and Birch, A. N. E. (2015). Modelling larval movement data from individual bioassays. Biometrical Journal, 57(3), 485-501.

Pewsey, A. (2000). The wrapped skew-normal distribution on the circle. Communications in Statistics - Theory and Methods, 29(11), 2459-2472.

Pewsey, A., Neuhäuser, M., and Ruxton, G. D. (2013). Circular Statistics in R. Oxford University Press, Croydon.

Walter L. Deemer, D. F. V. (1955). Estimation of parameters of truncated or censored exponential distributions. The Annals of Mathematical Statistics, 26(3), 498-504.

Wang, F. and Gelfand, A. E. (2014). Modeling space and space-time direc- 
tional data using projected Gaussian processes. Journal of the American Statistical Association, 109(508), 1565-1580. 